\title{
Diacronie
}

Studi di Storia Contemporanea

$\mathrm{N}^{\circ} 19,3$ | 2014

Miscellaneo

\section{Laurismo e janismo a confronto}

Due casi di populismo del secondo dopoguerra in prospettiva comparata (1947-1961)

\section{Federico Robbe}

\section{Q OpenEdition}

\section{Journals}

\section{Edizione digitale}

URL: http://journals.openedition.org/diacronie/1626

DOI: 10.4000/diacronie.1626

ISSN: 2038-0925

\section{Editore}

Association culturelle Diacronie

\section{Notizia bibliografica digitale}

Federico Robbe, «Laurismo e janismo a confronto », Diacronie [Online], № 19, 3 | 2014, documento 6, Messo online il 01 septembre 2014, consultato il 01 mai 2019. URL : http://journals.openedition.org/ diacronie/1626 ; DOI : 10.4000/diacronie.1626 


\section{Diacronie}

\section{6/}

\section{Laurismo e janismo a confronto Due casi di populismo del secondo dopoguerra in prospettiva comparata (1947-1961)}

Federico ROBBE*

L'articolo affronta due esperienze di populismo accomunate da un contesto in fase di modernizzazione (Italia e Brasile) e da un rapporto tra il leader carismatico e la parte più povera e umile della popolazione. I due Paesi, infatti, nei primi anni del secondo dopoguerra costituivano due laboratori populisti di un certo interesse, alla luce dei profondi mutamenti che stavano attraversando. Achille Lauro e Jânio Quadros seppero porsi nel contesto locale e nazionale come outsider di successo - uno da destra, l'altro da sinistra - grazie a campagne elettorali bizzarre ma estremamente efficaci, a messaggi semplificatori, e a una chiara idea di popolo a cui fare riferimento.

\section{Genesi ed evoluzione di due populismi: laurismo a destra e janismo a sinistra, 1947-1952}

\footnotetext{
7 l rapporto con la politica dell'armatore Achille Lauro è sempre stato caratterizzato dal massimo pragmatismo. Vicino al regime durante il Ventennio mussoliniano, che fece largo uso delle sua navi, se ne allontanò con la Repubblica di Salò. Venne però catturato dagli Alleati per i suoi precedenti rapporti col fascismo, e una volta liberato, al termine della guerra, decise di allacciare rapporti col mondo politico per poter ricostruire la sua flotta. Così, infatti, avrebbe potuto avere le necessarie protezioni al suo impero economico, che rinasceva grazie al credito proveniente da Londra e a due navi americane comprate e convertite da portaerei in transatlantici.
} 
Achille Lauro cercò subito di entrare in contatto con la DC, senza disdegnare però formazioni anche lontane dalla sua storia personale, come i socialdemocratici, e perfino il PCI. Eppure tutti i partiti tradizionali diffidavano di lui. Diffidavano cioè di un self made man egocentrico e molto ricco, potenzialmente in grado di influenzare le decisioni di partito e le strategie da adottare grazie alla sua eccezionale disponibilità finanziaria.

L'unica forza politica che si dimostrò disponibile a interloquire con Lauro fu il Fronte dell'Uomo qualunque di Guglielmo Giannini ${ }^{1}$, fondatore di un partito nato nel 1946 continuando l'esperienza del movimento omonimo sorto l'anno prima. Si trattava di una realtà ancora allo stadio magmatico, che aveva preso piede in massima parte nel Meridione; quindi la possibilità di avere dalla propria parte un outsider come Lauro fu percepita positivamente nella lotta ai politici di professione.

L'incapacità organizzativa all'interno dell'UQ e la non chiara posizione nel dibattito internazionale finirono per esaurire presto la spinta propulsiva del partito. E ad aggravare la situazione intervenne il dissidio con Lauro sul destino del governo De Gasperi, rispetto al quale Giannini si schierò curiosamente con le sinistre pur di far cadere l'esecutivo dello statista trentino nel 1947. Per Lauro, invece, non aveva alcun senso abbandonare il governo al suo destino, e in questo frangente dimostrò capacità tattiche ignote al commediografo fondatore dell'UQ, tant'è che intuì subito che il Partito nazionale monarchico di Alfredo Covelli poteva offrire maggiori spazi. Decise quindi di iscriversi al PNM, appianandone i debiti, e venne contestualmente eletto presidente. Restava tuttavia interessato a dialogare con la DC, non foss'altro che per la sua spropositata ambizione e per il potere decisionale dello Scudo Crociato ${ }^{2}$.

Oltre all'abilità nel leggere il corso degli eventi, unita a una certa spregiudicatezza, in questi primi anni del secondo dopoguerra emersero almeno altri tre aspetti che avrebbero segnato la lunga parabola politica del Comandante (nome con cui era conosciuto Lauro in ragione della sua attività imprenditoriale): il rapporto simbiotico, e spesso interessato, coi monarchici; l'acceso anticomunismo; l'appello al popolo in chiave leaderistica, con una totale identificazione tra il partito e il capo, anche in virtù delle ingentissime risorse economiche di cui questi disponeva. Basti pensare alla proprietà del giornale «Roma», assai letto a Napoli3, e alla carica di presidente del

\footnotetext{
${ }^{1}$ Su Giannini e il qualunquismo si vedano: SETTA, Sandro, L'Uomo qualunque, 1944-1948, Roma-Bari, Laterza, 2005; GIANNINI Guglielmo, La folla. Seimila anni di lotta alla tirannide, introduzione di ORSINA, Giovanni, Soveria Mannelli, Rubbettino, 2002.

2 UNGARI, Andrea, «Prima e oltre Berlusconi. Il caso Achille Lauro», in Rivista di politica, 2, 2011, pp. 103-118; p. 105.

3 Sulle vicende che portarono il «Roma» ad essere di proprietà di Achille Lauro si veda CAPRARA, Massimo, I Gava, Milano, Feltrinelli, 1975, pp. 34-35.
} 
Napoli calcio, utilizzata il più delle volte in chiave propagandistica per parlare direttamente al "suo" popolo4.

Del resto, Gioacchino Volpe nel luglio 1949 aveva previsto le possibili derive del "laurismo" proprio alla luce della simbiosi tra la proprietà personale di Lauro e il partito. In questi termini tentava di mettere in guardia Covelli:

Chi vuole mettere in dubbio le benemerenze e le capacità del comm. Lauro? Di uomini come lui, gran fortuna per un paese ne avesse cento! Ma veder cumulate nella stessa persona le qualità e le attività di un capo di partito, finanziatore del medesimo (e non occulto finanziatore, ma palesissimo, direi ufficiale finanziatore, con tutti i segni di riconoscimento di tal qualità) e grande uomo d'affari mi fece una certa impressione ${ }^{5}$.

Com'è stato rilevato sia in sede storiografica che politologica, il populismo presenta una intrinseca difficoltà ad essere posizionato lungo l'asse destra-sinistra ${ }^{6}$. E questo accade in ragione della vaghezza dell'appello al popolo, di volta in volta interpretato in chiave diversa da attori molto differenti tra loro ${ }^{7}$. Resta il fatto che lo spazio politico liberatosi dopo la crisi dell'Uomo qualunque, complice la contenuta forza dei neofascisti del MSI, era a destra, tant'è che Lauro non ha esitato a cavalcare battaglie populiste orientate appunto a destra, su tutte il legittimismo monarchico e il richiamo alla comunità organica di Napoli e al Regno del Sud contro Roma e il Nord. Un organicismo che ruotava attorno ad un rapporto privilegiato col "popolino" e con le classi subalterne. L'acceso anticomunismo e la diffidenza verso la DC contribuirono ad alimentare il consenso da destra, soprattutto nelle elezioni locali, e in misura minore anche in quelle nazionali.

La vicenda di Jânio Quadros, classe 1917, reca in sé numerosi aspetti contigui all'esperienza laurina. Non per formazione o per livello di istruzione, dato che è stato avvocato e docente universitario, bensì per lo stile politico e per la capacità di entrare direttamente in contatto con il popolo. Se nello scenario italiano schierarsi a destra

\footnotetext{
4 UNGARI, Andrea, op. cit., p. 108.

5 DE NAPOLI, Domenico, Il movimento monarchico in Italia dal 1946 al 1954, Napoli, Loffredo, 1980, pp. 59-60. Si veda anche ZULLINO, Pietro, Il Comandante. La vita inimitabile di Achille Lauro, Milano, SugarCo, 1976, pp. 59-61.

${ }^{6}$ ZANATTA, Loris, «Il populismo. Sul nucleo forte di un'ideologia debole», in Polis, 2/2002, p. 264.

7 Sull'eterogeneità del populismo nelle sue diverse manifestazioni concrete si vedano TAGGART, Paul, Il populismo, Troina, Città Aperta, 2002, p. 15. Sulla varietà di personaggi e contesti accomunati dall'accusa di populismo molto utile anche TARCHI, Marco, L'Italia populista. Dal qualunquismo ai girotondi, Bologna, Il Mulino, 2003, pp. 13-33.
} 
appariva la scelta più conveniente per Lauro, nel caso brasiliano la situazione era speculare. Il Partido Comunista Brasileiro (PCB) era stato bandito nel 1947, e la peculiarità del contesto paulista, caratterizzato da una fortissima e vorticosa modernizzazione industriale, aveva creato ampi settori di lavoratori in cerca di referenti politici. Collocarsi tra il centro e la sinistra, dunque, era per Jânio Quadros l'opzione ottimale. Un'opzione che univa un populismo "di classe" a un certo afflato moralizzatore ben identificato nella scopa, scelta come simbolo delle sue campagne elettorali, e brandita con forza da Quadros nei suoi comizi.

I due leader sono accomunati dalla ricerca di sponde politiche lungo tutto lo spettro parlamentare, il che rendeva il loro operato plasmato più dal pragmatismo che dall'ideologia. Così riuscirono a sfruttare al massimo il loro appeal di outsider estranei al sistema. Tra il 1945 e il 1947 Achille Lauro cercò contatti anche col PCI, prima di aderire all'Uomo qualunque e poi al Partito nazionale monarchico; Jânio Quadros iniziò la sua carriera con il Partido Democrata Cristão (PDC) e il Partido Socialista Brasileiro (PSB), ossia con un'alleanza tra nazionalconservatori e socialdemocratici. Da metà anni Cinquanta abbracciò invece partiti più orientati a sinistra, e perciò più sensibili alle richieste dei lavoratori.

Prima di candidarsi alla guida della città, Quadros era stato eletto all'assemblea legislativa del 1950, e in quell'occasione si era fatto notare per concretezza e realismo: era infatti riuscito ad attrarre voti da sinistra e a coinvolgere le organizzazioni di lavoratori grazia alla sua difesa degli scioperi e al suo sostegno ai movimenti per la pace contro il conflitto coreano ${ }^{8}$. Egli ricoprì la carica di sindaco (prefeito) di São Paulo (sostenuto da PDC e PSB) tra il 1953 e il 1954, e poi fu governatore dello Stato (195559), senza l'apporto del PDC, e per un breve periodo Presidente del Brasile (da gennaio ad agosto 1961), sostenuto stavolta dall'União Democrática Nacional (UDN) e da alcuni partiti conservatori, tra cui il PDC, ma non dal PSB. Tuttavia egli rimase sempre un indipendente e un personaggio sui generis.

Insomma, la sua fu una parabola politica non del tutto lineare. Come nel caso di Lauro, il messaggio era lui stesso; era il suo modo di fare politica e di incarnare "il nuovo", più per motivi anagrafici che professionali. L'ideologia politico-partitica di riferimento era sostanzialmente secondaria. Contava invece come il leader si rapportava alle masse, in che misura ne portava le istanze a livello regionale e nazionale, e soprattutto come risolveva i loro problemi. Anche in prima persona.

8 CHAIA, Vera, A liderança política de Jânio Quadros (1947-199o), Ibitinga, Humanidades, 1991, pp. 59-62. 
In particolare, è il legame con un certo tipo di popolo ad essere un fattore ben presente in entrambi. Possiamo dire che Quadros, a differenza dell'armatore italiano, si situa sullo spettro politico come un populista "di sinistra", pur non essendo organico ad alcun partito. Il suo era un populismo che identificava «la volontà del popolo con la giustizia e la morale» ${ }^{9}$, dunque con un'accezione più di classe e moralistica assai più che comunitaria, come era invece quella laurina.

Va sottolineato che i due contesti in cui agirono i leader populisti erano molto diversi dal punto di vista socioeconomico. Nel periodo compreso tra il 1945 e il 1960, il settore industriale crebbe a un tasso medio del 9,5\% in Brasile; e nel 1959 il 50\% delle industrie del Paese si trovava nello Stato di São Paulo, principalmente nella capitale o nelle immediate vicinanze. Tra il 1950 e il 1970, inoltre, il capoluogo paulista triplicò il numero degli abitanti, passando da 2 a 6 milioni $^{10}$. La città fu dunque caratterizzata da uno sviluppo che la rendeva il motore della crescita brasiliana postbellica. Uno scenario molto diverso quantitativamente e qualitativamente rispetto a Napoli, alle prese con gravi problemi di sopravvivenza e con un ruolo certo minore rispetto a quello giocato da São Paulo nello scenario nazionale. Dal punto di vista economico, nel contesto napoletano vi era una struttura dualistica che rispecchiava ed estremizzava quella nazionale: un limitato numero di fabbriche di grandi proporzioni (l'acciaieria di Bagnoli su tutte) e il 90\% di imprese con meno di 5 addetti. In più, a causa dei danni causati dai bombardamenti e dei provvedimenti governativi volti a rimborsare il $75 \%$ dei lavori per ogni abitazione, iniziò a prosperare una folta schiera di speculatori edilizi che sovrastimavano i danneggiamenti. Speculatori che poi furono in prima linea negli anni dell'amministrazione del Comandante Lauro e anche dopo ${ }^{11}$. In generale, è in questi anni che si delinea un modello di governo del territorio fortemente dipendente dall'intervento statale.

Nel 1951, la popolazione attiva impegnata nel settore industriale era il 10,9\%, a fronte del $16,2 \%$ a livello nazionale e dell' $8,5 \%$ nel Sud. Napoli, dunque, restava in una posizione leggermente più avanzata rispetto al resto del Meridione, tuttavia né la Cassa

\footnotetext{
9 Si veda l'intervento di HERMET, Guy, «Il populismo come concetto», in Diorama : Cos'è il populismo, 313, 1/2013, pp. 11-19; p. 12.

10 FONTES, Paulo, «Populism "from below": Working-class Neighborhoods and the Leadership of Jânio Quadros in São Paulo, Brazil», paper presentato al convegno internazionale Populism: a historiographic category?, Università degli Studi di Firenze, 8-10 aprile 2014, pp. 1-2; COLISTETE, Renato, Labour Relations and Industrial Performance in Brazil: Greater São Paulo, 1945-196o, Houndmill, Palgrave, 2001, cap. 1.

${ }^{11}$ Per quanto nell'immaginario collettivo Lauro sia associato alla speculazione edilizia, esistono studi secondo cui i danni maggiori sono stati fatti negli anni successivi, con l'amministrazione Gava, si veda DEMARCO, Marco, L'altra metà della storia. Spunti e riflessioni su Napoli da Lauro a Bassolino, Napoli, Guida, 2007, pp. 39-46.
} 
del Mezzogiorno né gli albori del boom economico mutarono di molto la sua collocazione. Nel 1961, infatti, al termine dell'esperienza laurina, la quota di occupati nel settore industriale era il 12,4\%, mentre a livello nazionale si attestava al 18,6\%. E se l'agricoltura vedeva progressivamente diminuire i propri addetti, non si verificavano incrementi di rilievo nell'ambito dei servizi (spesso peraltro di natura clientelistica e parassitaria), né, come si è visto, in quello industriale ${ }^{12}$.

Mentre il contesto paulista era più segnato dalla modernizzazione industriale, quello napoletano vedeva un periodo di mutamento al contempo politico-istituzionale e urbanistico, a causa sia dell'annunciata "rivoluzione" laurina contro la politica tradizionale, sia della speculazione edilizia. Quadros e Lauro, dunque, trovarono terreno fertile in società sulla via, più o meno accidentata, della modernizzazione: un carattere ricorrente del populismo, come ha messo lucidamente in evidenza Isaiah Berlin $^{13}$. Conviene ora esaminare più nel dettaglio le esperienze dei due leader populisti nei rispettivi contesti, per poter cogliere i tratti in comune e le specificità di ciascuno.

\section{Lo stile e le campagne elettorali di Achille Lauro: meridionalismo e marketing leaderistico, 1952-58}

Le elezioni amministrative del 1952 furono un importante test per Achille Lauro, che si candidò a sindaco di Napoli con il Partito nazionale monarchico. Due sono gli aspetti più interessanti della campagna elettorale: il primo riguarda le tecniche di marketing estremamente innovative, e il secondo è sui contenuti, fortemente antiromani e "sudisti". Le tecniche di propaganda sperimentate da Lauro sono un tassello imprescindibile per comprendere l'approccio orientato al leader. Il Comandante voleva instaurare con ciascuno un rapporto speciale, diretto, senza le logoranti dinamiche tipiche dei partiti di massa. Agli occhi dei suoi sostenitori, ha notato Tarchi, Lauro era

la negazione totale dell'invisa classe politica tradizionale: la rozza ma efficace concezione del marketing elettorale a cui ispira le sue campagne, distribuendo pacchi di pasta, zucchero, scarpe spaiate e banconote da mille lire tagliate a metà,

\footnotetext{
${ }_{12}$ ALLUM, Percy, Potere e società a Napoli nel dopoguerra, Torino, Einaudi, 1975, pp. 39-53; CARDILLO, Enrico, Napoli, l'occasione postindustriale. Da Nitti al piano strategico, Napoli, Guida, 2006, pp. 35-39.

${ }^{13} \mathrm{Si}$ veda in proposito ZANATTA, Loris, Il populismo. Sul nucleo forte di un'ideologia debole, cit., p. 265.
} 
promettendo il completamento del dono e elezione avvenuta, non fa che rafforzare questa sensazione ${ }^{14}$.

Quindi i metodi utilizzati si basavano su promesse ed elargizioni materiali, volte a soddisfare nell' immediato i bisogni dei napoletani.

L'organizzazione e la tecnica propagandistica di Lauro erano totalmente incentrate sul leader, e il partito era del tutto secondario, se non per i vaghi riferimenti alla monarchia. Era un qualcosa che lo stesso Lauro teneva in secondo piano, perché prima di tutto i consensi andavano a lui. Secondo Antonio Ghirelli, Lauro spese 600 milioni per le sole elezioni amministrative, facendo largo uso di «enormi gigantografie col suo ritratto ed il simbolo monarchico (Stella e Corona)». Ma soprattutto distribuì «agli elettori più bisognosi maccheroni, olio d'oliva, scarpe spaiate, pagando loro, talvolta, addirittura le polizze per gli oggetti impegnati al monte di pietà»15. Il filogovernativo «Corriere della Sera» riportava addirittura la cifra di 1 miliardo di lire per tutto il Meridione, dato che i manifesti dei monarchici erano i più diffusi e i più costosi. $\mathrm{Al}$ quotidiano di via Solferino si poteva cogliere una viva preoccupazione per la popolarità di Lauro ${ }^{16}$. L'insistenza sulla sua persona e sul soddisfacimento dei bisogni - due elementi percepiti nell'immaginario collettivo come un tutt'uno - erano paradigmatici di questo approccio. Elementi che hanno caratterizzato anche la strategia elettorale di Quadros, come si vedrà.

Il paternalismo di Lauro e la sua tecnica di marketing leaderistica sfruttarono la situazione socio-economica disastrosa della città, che alimentò le illusorie speranze dell'elettorato. Il Comandante decise di puntare tutto sugli annunci di opere pubbliche, che poi non di rado furono costruite grazie a finanziamenti personali.

Per Lauro dunque non è eccessivo parlare di un vero e proprio "culto della personalità":

la continua "assunzione di responsabilità" che Lauro compie - con la propria biografia di self made man, con l'evidenziazione della propria capacità di superare con l'iniziativa personale gli ostacoli burocratici, fino al "gallismo sbrigativo e ostentato", ulteriore riprova di un'immagine vitalistica - risulta in definitiva una caratteristica del mito laurino di cruciale importanza; una caratteristica ben accetta ad ampi settori sociali privi di tradizioni democratiche e di consuetudine alla

14 TARCHI, Marco, op. cit., p. 96.

15 GHIRELLI, Antonio, Achille Lauro, Napoli, Gaetano Macchiaroli, 1992, p. 119, cit. in UNGARI, Andrea, op. cit., p. 113 .

${ }_{16}$ NEGRO, Silvio, Non vuol vivere di solo pane il Mezzogiorno, in «Corriere della Sera», 22 maggio 1952. 
partecipazione politica attiva, e propenso di conseguenza alla delega e all'identificazione con un leader con cui vi è comunque notevole omogeneità culturale $^{17}$.

La mobilitazione di buona parte dell'elettorato trovava una delle sue ragioni principali nella certezza che Lauro fosse diverso rispetto ai politici tradizionali. Che Lauro, in qualche modo, fosse come loro, uno del sottoproletariato; o comunque che lo fosse stato prima di affermarsi come imprenditore. Un messaggio di possibilità e di speranza, connesso alla sua storia personale e a un certo disprezzo per le regole, si levava con la sua battaglia contro Roma e a favore del Sud. La miscela di sentimenti populisti e storia di successo personale diede ottimi frutti nel 1952: il 40\% dei consensi e 117.00o preferenze. Tanto che molti giornali scrissero che aveva vinto il laurismo più che il Partito nazionale monarchico. Non a caso il PNM era passato da 44.390 voti del 1946 agli oltre 147.800 del 1952 essenzialmente grazie al Comandante. Per sottolineare come l'avvento di Lauro venisse auspicato trasversalmente dai cittadini, e fosse visto realmente come una novità dirompente, basti ricordare che il precedente sindaco democristiano non venne neanche confermato consigliere comunale ${ }^{18}$.

Alle elezioni politiche del 1953 il sindaco di Napoli si dimostrò intenzionato a esportare il laurismo al Nord e condizionare da destra la DC. Gli industriali settentrionali, però, risposero in modo decisamente negativo alle proposte di Lauro ${ }^{19}$. Varie erano le ragioni all'origine del rifiuto della grande borghesia del Nord: il rilancio della politica nazionalista venne associato all'esperienza fascista; nel partito di Lauro, inoltre, vi erano alcuni personaggi impresentabili perché troppo legati al Ventennio fascista. In più, la vicinanza di Confindustria (allora presieduta dall'armatore Angelo Costa) ai liberali e alla $\mathrm{DC}^{20}$ affossò definitivamente il progetto di Lauro. Così, il partito fu in qualche modo costretto a restare un partito locale, al massimo con ambizioni regionali o meridionali.

Anche nella campagna del '53 furono utilizzate tecniche di propaganda incentrate sulla personalizzazione della leadership, con un massiccio impiego di risorse e la distribuzione di cibo.

17 VIGILANTE, Riccardo, Politica, sentimenti e soldi: la campagna elettorale del 1953, in CHIANESE, Gloria (a cura di), Il silenzio della ragione. Politica e cultura a Napoli negli anni Cinquanta, Napoli, ESI, 1994, pp. 75-76.

${ }^{18}$ Si tratta di Domenico Moscati, si veda CAPRARA, Massimo, op. cit., p. 31.

${ }^{19}$ Si veda COLARIZI, Simona, Storia del novecento italiano, Milano, Rizzoli, 200o, p. 348.

${ }^{20}$ DE NAPOLI, Domenico, op. cit., p. 125. Si veda l'articolo di WOLLEMBORG, Leo, in «Washington Post», 9 maggio 1953, riportato interamente in ZULLINO, Pietro, op. cit., pp. 6569. 
Il sindaco riuscì a riportare in ambito politico-propagandistico l'approccio agli affari, immediato, orientato al risultato e spesso fin troppo sfacciato. Si perpetuò il ricorso alla distribuzione di generi alimentari di prima necessità, sicché all'inizio della campagna elettorale furono aperte mense popolari nei quartieri più disagiati, e a quelle mense poteva accedere chiunque presentasse la tessera del PNM. Gli attivisti monarchici, si legge sul «Corriere della Sera», «girano sempre con tessere del partito pronte in tasca, ed esse trovano collocamento proprio nei quartieri popolari». Già l'anno precedente, le elezioni amministrative avevano rivelato la validità di questo argomento, dato che la plebe napoletana aveva mantenuto la parola coi distributori di pasta e di riso ${ }^{21}$. In ogni caso, il fenomeno-Lauro continuò: sulla scia della sorprendente affermazione nelle amministrative, il suo partito ottenne nel 1953 il 6,8\% dei consensi, più che raddoppiando il risultato di cinque anni prima. L'armatore collezionò quasi 183.00o preferenze, ma poi fu costretto a scegliere tra il parlamento e il Comune di Napoli, optando per la carica di sindaco.

La coalizione guidata dalla $\mathrm{DC}$, com'è noto, prevalse ma non riuscì a far scattare il premio di maggioranza per poche decine di migliaia di voti. E da questo momento i vertici democristiani iniziarono a rendersi conto che il peso elettorale di Lauro e Covelli non poteva più essere trascurato. Tuttavia gli attriti tra i due leader del PNM iniziarono ad essere sempre più frequenti. Lauro sosteneva che il partito fosse suo ${ }^{22}$, dato che se l'era comprato appianando i debiti pregressi, e quindi adesso lo poteva gestire come voleva. Del resto, il PNM era "cucito" interamente addosso a Lauro, specialmente a Napoli, dove lo spirito revanscista associato alla Corona acquisiva una qualche visibilità solo attraverso l'impegno del Comandante. Questa eccessiva personalizzazione non veniva però vista di buon occhio dal segretario Covelli, sicché presto i due arrivarono a una rottura insanabile, con Lauro deciso a continuare la sua scalata al potere. Ciò avvenne nel 1954, con la nascita del Partito monarchico popolare (PMP). A Covelli rimaneva il PNM, destinato ormai all'irrilevanza politica senza la disponibilità finanziaria dell'armatore sorrentino ${ }^{23}$.

Le amministrative del 1956 suggellarono ancora la popolarità del Comandante con un successo impressionante. Il numero di preferenze arrivò a circa 242.000 (su oltre

${ }^{21}$ NEGRO, Silvio, I punti deboli di Lauro nella campagna elettorale in Campania, in «Corriere della Sera», 10 maggio 1953.

${ }^{22}$ LOMARTIRE, Carlo Maria, 'O Comandante. Vita di Achille Lauro, Milano, Mondadori, p. 110.

23 BALDONI, Adalberto, La destra in Italia 1945-1969, Roma, Pantheon, 2000, p. 395; ROSSI, Mario Giuseppe, «Il governo Scelba tra crisi del centrismo e ritorno anticomunista», in Italia contemporanea, n. 197, dicembre 1994, p. 795; COLARIZI, Simona, op. cit., pp. 348-349. Per un inquadramento più generale si vedano ALLUM, Percy, op. cit., pp. 354-360; GRIBAUDI, Gabriella, Mediatori. Antropologia del potere democristiano nel Mezzogiorno, Torino, Rosemberg e Sellier, 1980. 
276.00o voti di lista per il PMP), quasi triplicando il lusinghiero risultato della precedente tornata elettorale. Il partito di Lauro ottenne il 51\%, e questo gli consentiva di governare la città senza la DC.

Durante il secondo mandato continuò a intervenire direttamente per risolvere i problemi del "popolino", dei disoccupati e della folla dei senza tetto. Stando a quanto scriveva «L’Espresso», si adoperò personalmente per far fronte alle richieste:

Lauro accontenta tutti: direttamente, se può, facendosi sentire all'INA-casa, ai cantieri di lavoro, all'ufficio di collocamento. Alla ditta Trezza, appaltatrice delle imposte di consumo, ha ordinato di investire, contro maggiorazione dell'aggio di riscossione, settecento milioni nel "Villaggio Lauro". Alla Rinascente ha concesso di elevare un edificio di sedici piani su un suolo comunale a patto che assumesse cento ragazze raccomandate da lui. Alla società di assicurazioni "La Cattolica" ha fatto pagare lo stesso strappo al regolamento edilizio con venti milioni per le opere di assistenza. Sono generosità fruttuose, intendiamoci, che rendono il mille per cento, quadruplicando il valore dei suoli. Ma Lauro, che non crede nel fisco, nei partiti, nella democrazia, pensa che non c’è altra via per cavare denaro ai ricchi e alleviare i poveri $^{24}$.

Il trionfo popolare del '56 spronò Lauro a esportare il suo modello non tanto al Nord - come in precedenza - ma soprattutto nel Mezzogiorno, ponendosi come «campione delle genti meridionali». Nel 1957, per le elezioni regionali sarde, il Comandante tentò di sfruttare la televisione, uno strumento che sarebbe diventato oggetto di numerose riflessioni sul populismo mediatico nei decenni successivi. Avendo iniziato le trasmissioni solo pochi anni prima, nel 1954, la tv era ancora ad uno stadio molto embrionale sia in termini di diffusione sul territorio - e a maggior ragione al Sud - che di "confidenza" tributata dagli italiani. Tuttavia, Lauro aveva intuito la pervasività del mezzo, immediato come il suo stile politico; semplice, efficace, spesso anche rozzo. Ma comunque senza mediazioni. E così immaginò di poter apparire sugli schermi del Sud per lanciare la riscossa del Meridione. Pensò quindi di chiedere al ministero delle Poste l'installazione di una televisione privata, ma l'istanza fu rigettata e la faccenda si chiuse, nonostante il ricorso di Lauro al Consiglio di Stato ${ }^{25}$. In Sardegna il risultato fu in ogni caso eccezionale: il PMP ottenne il 9\% dei consensi ${ }^{26}$.

24 Articolo di Cesare Zappulli apparso in «L'Espresso» il 9 ottobre 1955, cit. in ZULLINO, Pietro, op. cit., p. 85 .

${ }^{25}$ L'idea continuò a interessare e stimolare Lauro anche nei decenni successivi. Fu lui infatti - a 89 anni - a fondare il Canale 21, nel 1976, con Andrea Torino. Era la prima televisione privata italiana. L'esperienza, tuttavia, durò poco a causa dell'ostilità dei partiti che non ammettevano 
Dopodiché iniziarono le ispezioni sulla gestione finanziaria del Comune di Napoli da parte della DC di Fanfani, particolarmente preoccupato dell'avanzata laurina. E fu facile trovare irregolarità macroscopiche di fronte alle quali Lauro si dimise, sperando così di salvare il rapporto col suo popolo. Ma la mossa non venne apprezzata dall'elettorato napoletano; anzi, venne interpretata come un tradimento proprio ai danni degli ultimi, ossia di quelli che avevano creduto in lui fin da subito.

Col 1958 la parabola politico-amministrativa di Lauro può considerarsi conclusa, eccezion fatta per un clamoroso ritorno da sindaco nel 1960. Ma fu un'esperienza di pochi mesi. Nonostante quasi 207.000 voti di preferenza, l'anno successivo, nel marzo '61, i consiglieri democristiani votarono contro il bilancio, costringendo così l'armatore a farsi da parte. Inoltre, pochi mesi dopo, ben 7 consiglieri monarchici passarono alla DC. Non solo il partito del Comandante perdeva così la maggioranza relativa, ma oltretutto ciò avveniva in circostanze rocambolesche, con uomini di fiducia che passavano al partito per anni combattuto (ma anche corteggiato) dallo stesso Lauro ${ }^{27}$.

\section{Ascesa del janismo: appello al "popolo-classe", moralizzazione della vita politica e soluzione immediata dei problemi, 1952-1961}

Diversi sono i tratti in comune tra Lauro e Quadros. Il carismatico leader brasiliano riuscì con successo a intercettare le istanze degli ultimi, dei più poveri, delle periferie. Di tutti quelli che si trovavano in qualche modo abbandonati nel vorticoso processo di urbanizzazione e industrializzazione della città. Nel caso del janismo, sin dalla corsa di Quadros a deputato al parlamento brasiliano e poi ancora di più con la candidatura a sindaco, emersero alcuni tratti distintamente populisti. Il passaggio problematico della modernizzazione in corso andava ad innestarsi in un contesto peraltro complesso come quello latinoamericano. Dove le analisi "strutturaliste" del populismo sono state ormai da diversi anni integrate da interpretazioni di più ampio respiro, volte a cogliere caratteri sedimentati, come per esempio un certo organicismo di matrice religiosa. Caratteri insomma che affondavano le proprie radici ben prima degli anni di intensa modernizzazione postbellica ${ }^{28}$.

interferenze in un ambito così delicato come l'informazione. Si vedano LOMARTIRE, Carlo Maria, op. cit., p. 188; DELLA RAGIONE, Achille, Achille Lauro superstar. La vita, l'impero, la leggenda, Napoli, Guida, 2003, p. 79

${ }^{26}$ LOMARTIRE Carlo Maria, op. cit., pp. 153-154.

27 SETTA, Sandro, op. cit., pp. 236-237.

${ }^{28}$ Sul populismo in America Latina si veda, tra i vari contributi, ZANATTA, Loris, «Il populismo in America Latina. Il volto moderno di un immaginario antico", in Filosofia politica, 3/2004, pp. 377-389. Sul populismo brasiliano si vedano FERREIRA, Jorge, O populismo e sua história: 
Quadros fu particolarmente abile nell'organizzare centinaia di comitati elettorali a partire dalle cosiddette Sociedades Amigos de Bairro (SAB), ovvero delle associazioni degli amici di quartiere emerse alla fine degli anni Quaranta nei quartieri più disagiati ${ }^{29}$. Spesso erano associazioni che esprimevano le difficoltà di chi faticava ad adattarsi al nuovo contesto, e chiedevano il più delle volte anche solo piccoli miglioramenti. Tramite innumerevoli lettere giungevano a Jânio Quadros richieste per rispondere ai bisogni di prima necessità: illuminazione pubblica, telefoni in punti strategici, strade asfaltate ${ }^{30}$.

Alla base del janismo c'era proprio questo rapporto diretto con gli abitanti dei quartieri più poveri, conquistati anche grazie ai ripetuti appelli all'onestà e alle critiche alla corruzione assai diffusa nel Paese. Questo accento moralizzatore fu certamente uno degli elementi decisivi nella sua elezione a sindaco.

Tra i quartieri più poveri era assai diffusa l'idea che Quadros fosse un politico diverso, ma allo stesso tempo simile alla maggior parte dell'elettorato31'. Del resto, non è indispensabile che il leader appartenga alla medesima classe sociale del suo popolo. Tant'è che non accade quasi mai. È invece decisivo che il leader sia in grado di «inscenare in forma credibile un simulacro di democrazia diretta, un contatto istantaneo, privo di mediazioni astratte e impersonali, quasi intimo e confidenziale con “il popolo" ${ }^{32}$. E Quadros, eccentrico outsider della politica brasiliana, riuscì a stabilire uno stretto rapporto fiduciario coi lavoratori. Lo dimostrò durante la campagna elettorale e nel corso del biennio alla guida della città. A contendergli l'elezione vi era il favoritissimo Francisco Antônio Cardoso, che era supportato da sette partiti di diverso orientamento, con una forte impronta nazionalista e conservatrice: l'União Democrática Nacional (UDN), il Partido Social Progresista (PSP), ma anche formazioni più orientate a sinistra come il Partido Social Democrático (PSD) e il Partido Trabalhista Brasileiro (PTB). Quadros grazie al lavoro capillare delle SAB riuscì a imporsi con un impressionante $65,8 \%$.

Una volta diventato sindaco, il suo rapporto con le associazioni si fece ancora più intenso. Quadros interveniva personalmente per rispondere alle petizioni che incessantemente arrivavano sulla sua scrivania. E quando risolveva alcuni problemi

debate e crítica, Rio de Janeiro, Civilização Brasileira, 2001; WEFFORT, Francisco, $O$ populismo na política brasileira, Rio de Janeiro, Paz e Terra, 1980.

29 HOLSTON, James, Insurgent Citizenship. Disjunctions of Democracy and Modernity in Brazil, Princeton, Princeton University Press, 2008, p. 237.

$3^{\circ}$ FONTES, Paulo, op. cit., p. 14.

${ }^{31}$ CONNIFF, Micheal, Brazil's Populist Republic and Beyond, in ID (a cura di), Populism in Latin America, Tuscaloosa, University of Alabama Press, 2012, p. 57.

32 ZANATTA, Loris, «Io, il popolo. Note sulla «leadership» carismatica nel populismo latinoamericano», in Ricerche di Storia Politica, 3/2002, p. 438. 
come l'illuminazione pubblica o altri piccoli miglioramenti dello status del sottoproletariato, il sindaco stesso scriveva ai responsabili delle SAB per comunicare il risultato.

Un rapporto quindi senza mediazione alcuna, come avvenne anche dal punto di vista propagandistico. Appena un anno dopo l'elezione, infatti, Quadros decise di candidarsi a governatore dello Stato di São Paulo, e nel corso della campagna elettorale usò sapientemente la televisione per fare appello ai lavoratori33. Fu il primo politico brasiliano ad aver intuito la potenzialità del mezzo, proprio come Lauro avrebbe cercato di fare pochi anni dopo in Italia. Quadros sconfisse Ademar de Barres per poche migliaia di voti, pari all'1\%. Alla guida del governatorato continuò la sua opera di moralizzazione e di critica alla vecchia classe politica, che a lui, neanche quarantenne, appariva solo una fucina di privilegi e di corruzione.

La rapida ascesa di Quadros culminò con l'elezione a presidente della Repubblica. Sostenuto da varie liste ma principalmente dall'UDN, che in precedenza l'aveva osteggiato, venne eletto nell'ottobre 1960. Aveva solo 43 anni34. Le campagne elettorali furono ancora una volta basate sul personalismo e sul rapporto con le associazioni di lavoratori delle classi subalterne. Per quel che riguarda lo stile, Quadros proseguì con i suoi comportamenti anticonformisti. Aveva capito che i brasiliani volevano un politico diverso. E lui non esitava ad armeggiare con una scopa gigante nel corso dei comizi, e a portare con sé una gabbia con un topo mentre proclamava che da presidente «si sarebbe liberato di tutti i topi corrotti al governo»35. Aveva capito insomma che il popolo - soprattutto il "suo" popolo - aveva bisogno di una presidenza di rottura. E le urne gli diedero ragione, dato che conquistò il 48\% dei consensi con circa 5,6 milioni di voti. Oltre due milioni in più dell'altro candidato, il maresciallo dell'esercito Henrique Teixeira Lott.

Giunto al governo, però, Quadros non riuscì a tradurre concretamente un programma vago, troppo "contro" e poco propositivo, al di là degli accenti moralizzatori. In pochi mesi venne a crearsi una situazione di stallo tra lui e gli alleati in parlamento. Cause dell'incomunicabilità erano la genericità delle proposte, l'eccessiva enfasi sull'austerità fiscale, l'impatto negativo dello slancio moralizzatore sui costumi, e la sua ambigua condotta in ambito internazionale. Anche in politica estera, infatti, Quadros non riuscì a muoversi in maniera coerente: all'iniziò tentò di attirare gli aiuti economici degli Stati Uniti, ma poi cercò di intercettare i consensi di altri Paesi

33 CONNIFF, Micheal, op. cit., p. 57.

34 ROETT, Riordan, New Brazil, Washington D.C., Brookings Institution, 2010, p. 50.

35 CARDOSO, Fernando Henrique, The Accidental President of Brazil: a Memoir, Cambridge, Perseus Book Groups, 2006, p. 58. 
come l'Egitto di Nasser e la Cina di $\mathrm{Mao}^{36}$. Creando così confusione nell'elettorato e logoramento nei partiti della coalizione. L’esperienza alla guida del Paese durò solo pochi mesi: nell'agosto '61 Quadros rassegnò le dimissioni parlando di “forze terribili" che avrebbero cospirato contro di lui. Il governo passava al vice presidente Goulart (membro del PTB), dopo un iniziale veto dei militari.

Nel 1962 Quadros cercò di tornare alla guida dello Stato di São Paulo, ma venne sconfitto proprio da Ademar de Barres, il suo sfidante di pochi anni prima. A quel punto la parabola del janismo subì una battuta d'arresto. Un ulteriore decisivo elemento contribuì a decretarne la fine, ossia la decennale revoca dei diritti politici, decretata dal governo militare che prese il potere nel $1964^{37}$.

\section{Conclusione}

Il laurismo e il janismo tendono a configurarsi come due populismi distanti sullo spettro politico, almeno in termini di elettorato di riferimento e di concezione stessa di "popolo"38: più classista quella di Quadros, più organica quella di Lauro. Tuttavia ci sono due fattori che li accomunano: il primo è lo stile delle campagne elettorali, e il secondo è l'accento sulla soluzione immediata dei problemi. Lo stile di entrambi infatti fu sostanzialmente incentrato sul rapporto diretto con un leader carismatico e in qualche modo estraneo al circuito politico tradizionale, vuoi per provenienza (nel caso dell'imprenditore Lauro) vuoi per i gesti di rottura e per ragioni anagrafiche (la già citata scopa con cui armeggiava il trentacinquenne Quadros).

Oltretutto, nella loro permanenza al governo, soprattutto a livello locale, si distinsero per una capacità di empatia nei confronti del loro popolo di riferimento. Lauro utilizzò anche risorse finanziarie personali, sfruttò il sentimento monarchico e vagheggiò una comunità ideale meridionale. Quadros puntò sul decisionismo, sull'indipendenza dai partiti e sulla rete di associazioni di lavoratori. Entrambi erano personaggi indubbiamente eccentrici, interessati a una mobilitazione continua.

In altri termini - e veniamo al secondo elemento in comune - non avevano solamente un'ambizione plebiscitaria che si risolvesse nelle urne, in attesa della successiva tornata elettorale. Incarnarono piuttosto un populismo dove il rapporto col

${ }^{36}$ MENDES, Candido, Beyond Populism, Albany, State University of New York Press, 1977, p. 7; CONNIFF, Micheal, op. cit., p. 59.

${ }_{37} \mathrm{Si}$ veda URL: < http://www.biblioteca.presidencia.gov.br/english/former-presidents/janioquadros $>$ [consultato il 23 giugno 2014].

${ }_{38}$ Per un'analisi dell'appello al popolo come uno dei caratteri distintivi del populismo si vedano almeno TARCHI, Marco, op. cit., p. 17; ZANATTA, Loris, Il populismo. Sul nucleo forte di un'ideologia debole, cit., pp. 264-265; TAGGART, Paul, op. cit., pp. 151-157. 
popolo andava continuamente alimentato. Ciò accadeva tramite strumenti diversi: a Napoli con pacchi di pasta, beni di prima necessità, impieghi nella pubblica amministrazione, e per i più fortunati perfino una casa o un appalto; a São Paulo lampioni, strade asfaltate, telefoni pubblici. Più individuale il laurismo, più collettivo il janismo, ma entrambi orientati verso la soddisfazione immediata delle richieste. Immediata sia dal punto di vista per così dire procedurale (senza mediazioni), sia dal punto di vista temporale (subito).

Come ha notato acutamente Guy Hermet, quello che desidera il popolo è soprattutto la soppressione della distanza «che separa i suoi desideri personali o collettivi immediati dalla loro realizzazione, sempre differita in nome delle complicazioni dell'azione politica». Lauro e Quadros riuscirono perfettamente nell'intento. Era lo «sfruttamento sistematico del sogno in tempo reale»39, in opposizione ai compromessi, per forza di cose lunghi e farraginosi, della democrazia; e allo stesso tempo era un approccio diverso dal paradiso in terra promesso dai totalitarismi, anch'esso spostato in un futuro indefinito.

39 HERMET, Guy, op. cit., p. 14; JAGUARIBE, Hêlio, Problemas do desenvolvimiento latinoamericano, Rio de Janeiro, Isbe, 1967, p. 168. 


\section{* L'autore}

Federico Robbe ha conseguito il dottorato di ricerca presso l'Università degli Studi di Milano nel 2010. Tra le sue pubblicazioni vi è il volume: L’impossibile incontro. Gli Stati Uniti e la destra italiana negli anni Cinquanta, Milano, Franco Angeli, 2012 (finalista del premio Acqui Storia). Si occupa di storia dell'Italia repubblicana, in particolare nella sua dimensione internazionale, di populismo e di storia della destra. Attualmente (2014) sta lavorando a un progetto di ricerca dell'Università della Valle d'Aosta su Giulio Andreotti e le Regioni autonome di confine.

URL: < http://www.studistorici.com/progett/autori/\#Robbe >

\section{Per citare questo articolo:}

ROBBE, Federico, «Laurismo e janismo a confronto. Due casi di populismo del secondo dopoguerra in prospettiva comparata (1947-1961)», Diacronie. Studi di Storia Contemporanea, 29/09/2014,

URL:< http://www.studistorici.com/2014/09/29/robbe_numero_19/ >

\section{Diacronie Studi di Storia Contemporanea $\beta$ www.diacronie.it}

Risorsa digitale indipendente a carattere storiografico. Uscita trimestrale. redazione.diacronie@hotmail.it

Comitato di redazione: Jacopo Bassi - Luca Bufarale - Elisa Grandi - Deborah Paci - Fausto Pietrancosta - Matteo Tomasoni - Luca Zuccolo 